\title{
Importancia de la clasificación de obesidad en la tasa de erradicación de Helicobacter pylori
}

Acta Gastroenterol Latinoam 2021;51(1):7-9

Recibido: 04/12/2020 / Aceptado: 19/02/2021 / Publicado online: 22/03/2021 / https://doi.org/10.52787/vfoy2155

\section{Sr. Editor:} radores titulado "Erradicación de Helicobacter pylori en pacientes obesos pre-cirugía bariátrica", ${ }^{1}$ publicado en Acta Gastroenterol Latinoam 2020;50(1):40-44. Nos parece interesante que se centre en el uso de un esquema cuádruple como método de erradicación de Helicobacter pylori en pacientes obesos. Aun así, notamos que el estudio carece de una clasificación sobre obesidad, basado en ra puede influir en los resultados tanto del tratamiento como en la tasa de prevalencia de Helicobacter pylori en estos pacientes.

La eficacia del esquema cuádruple para erradicación del Helicobacter pylori está basada en consensos españoles y europeos; estos esquemas se modifican de acuerdo con la tasa de prevalencia de Helicobacter pylori y la resistencia antibiótica para escoger el tratamiento adecuado., ${ }^{2,3}$

Estudios demuestran que la tasa de prevalencia de $\mathrm{He}$ licobacter pylori aumenta de manera distinta en cada grado de obesidad. Se observa una prevalencia de Helicobacter pylori en personas sin obesidad en un $27,0 \%$,
Leímos el artículo publicado por Laudanno y colaboel índice de masa corporal (IMC), que de cierta mane-

a diferencia de personas con obesidad de grado I y grado II (IMC 34,6 $\pm 3,7 \mathrm{~kg} / \mathrm{m}^{2}$ ) en un $57,2 \% .{ }^{4,5}$ Debido a estas diferencias, se sugeriría que la eficacia del tratamiento se ve afectada por el índice de masa corporal del paciente. A mayor índice de masa corporal, mayor tasa de prevalencia y por tanto menor eficacia del tratamiento. Esta hipótesis se explora en un estudio donde la eficacia del tratamiento en personas sin obesidad es de un $85,3 \%$ y en personas con obesidad de un $55,0 \%$, por lo que es pertinente confirmar dichos resultados. ${ }^{6}$

El trabajo es interesante, pero sería pertinente que en estudios posteriores donde se evalúe la respuesta al tratamiento de Helicobacter pylori se considere presentar los resultados de acuerdo a los grupos generados por la IMC, para hablar acerca de la eficacia del tratamiento en pacientes obesos.

\section{Bryan Cuzcano Villalobos Kevin Quinteros Figueroa Jorge Osada Liy}

Universidad Privada San Juan Bautista. Chincha Alta, Ica, Perú

Conflictos de intereses. Los autores declaran no tener ningún conflicto de interés.

\section{Referencias}

1. Laudanno O, Ahumarán G, Thomé M, Gollo P, Khoury M. Erradicación del Helicobacter pylori en pacientes obesos pre-cirugía bariátrica. Acta Gastroenterol Latinoam. 2020;50(1):40-4.

Correspondencia: Bryan Cuzcano Villalobos Chincha Alta, Ica, Perú

Tel.: +51931902064

Correo electrónico: bryan.cuzcano@upsjb.edu.pe
2. Sanjurjo JL, Manauta JP, Hidalgo H, Geyne A. Eficacia y seguridad entre diferentes dosis de nitazoxanida + subcitrato de bismuto + lansoprazol para la erradicación de Helicobacter pylori. Rev Med Hosp Gen, Mex. 1999;62(3):172-5. 
3. Gisbert JP, Molina Infante J, Amador J, Bermejo F, Bujanda L, Calvet X, et al. IV Conferencia Española de Consenso sobre el tratamiento de la infección por Helicobacter pylori. 2016;39(10):697-721.

4. Arslan E, Atilgan H, Yavaşoǧlu I. The prevalence of Helicobacter pylori in obese subjects. Eur J Intern Med. 2009;20(7):695-7.
5. Zhang J, Chen Y, Chen W, Xu H, Wang H, Chen L, et al. Persistent infection of Helicobacter pylori affects weight loss in obese population compared with persistent negative: A case-control study based on healthy Chinese. Helicobacter. 2020;25(4):e12697.

6. Abdullahi M, Annibale B, Capoccia D, Tari R, Lahner $\mathrm{E}$, Osborn $\mathrm{J}$, et al. The eradication of Helicobacter pylo$r i$ is affected by body mass index (BMI). Obes Surg. 2008; 18(11):1450-4.

\section{Respuesta de los autores}

Bryan Cuzcano Villalobos y col. afirman que los pacientes obesos tienen mayor prevalencia de Helicobacter pylori, que esta aumenta con el grado de obesidad y que esta mayor prevalencia afecta la eficacia de los tratamientos de erradicación. Por otro lado, que el estudio carece de una clasificación de obesidad basada en el índice de masa corporal (IMC).

Respecto de la mayor prevalencia Helicobacter pylori en pacientes obesos, esto es muy controvertido ya que hay estudios que muestran que la prevalencia es menor, otros que muestran que es igual y otros que es mayor. Globalmente los estudios muestran una menor prevalencia Helicobacter pylori en los pacientes obesos. ${ }^{1,2}$ Sigue vigente la teoría que sugiere una correlación inversa entre la prevalencia de Helicobacter pylori y obesidad. Desde el pun-

Correspondencia: Dr Oscar Laudanno

Departamento de Gastroenterología, Instituto de Investigaciones Médicas Alfredo Lanari. Universidad de Buenos Aires, Argentina

Correo electrónico: oscarlaudanno@gmail.com to de vista fisiopatológico, la infección con Helicobacter pylori alteraría la secreción de grelina. Distintos estudios han mostrado que luego de la erradicación de Helicobacter pylori aumenta la secreción de grelina y los pacientes ganan peso. ${ }^{3,4}$ Los datos de prevalencia de Helicobacter pylori en pacientes obesos derivados para cirugía bariátrica de nuestro grupo son de alrededor del $62 \% .^{5}$

En este trabajo, todos los pacientes que estudiamos dentro del grupo con obesidad eran pacientes derivados para cirugía bariátrica y esto implicaba que los mismos reunieran los criterios aceptados por las distintas guías, es decir que tenían un IMC mayor de 35 con más de una comorbilidad o un IMC mayor de $40 .{ }^{6}$ No fue objetivo del estudio al que se hace referencia evaluar la eficacia del tratamiento de erradicación de acuerdo al grado de obesidad, sino comparar la erradicación de Helicobacter pylori entre pacientes derivados para cirugía bariátrica y pacientes con IMC normal.

Finalmente, está el tema de la eficacia de los tratamientos de erradicación de Helicobacter pylori en pacientes obesos. Esto, claramente, no se vincula a la mayor prevalencia. Si bien, como planteamos en nuestro estudio, es controvertido que los pacientes obesos tengan menor respuesta, nuestro grupo apoyado en el estudio publicado de referencia, así como en otro posterior, cree que esto es así y tenemos como hipótesis que se debe a que los pacientes obesos tienen alterada la farmacocinética y farmacodinamia de los antibióticos. Es por ello que, con un esquema ajustado al peso corporal, se mejoraron en forma significativa las tasas de erradicación: 86,3\% (95\% 
CI: 74,3-93,2)] en el esquema ajustado al peso corporal y $66,1 \%(95 \%$ CI: $52,6-77,3)$ en el grupo tratado con dosis fijas ( $p=0,02890)$, RR 1,31 (95\% CI: 1,05-1,63). Ambos grupos fueron tratados con un esquema cuádruple concomitante de catorce días de duración. En este estudio, más del 30\% de los pacientes tenían un IMC > 40 .

\author{
Oscar Laudanno ${ }^{1}$ \\ Gabriel Ahumarán ${ }^{2}$ \\ Marcelo Thomé ${ }^{3}$ \\ Pablo Gollo ${ }^{2}$ \\ Marina Khoury ${ }^{4}$ \\ ${ }^{1}$ Departamento de Gastroenterología, Instituto de Investigaciones \\ Médicas "Alfredo Lanari", Universidad de Buenos Aires. Ciudad \\ Autónoma de Buenos Aires, Argentina. \\ 2 Sección Gastroenterología, Hospital Bocalandro. Tres de \\ Febrero, prov. de Buenos Aires, Argentina. \\ ${ }_{3}^{3}$ Departamento de Gastroenterología, Hospital Eva Perón. San \\ Martín, prov. de Buenos Aires, Argentina. \\ ${ }^{4}$ Docencia e Investigación, Instituto de Investigaciones Médicas \\ "Alfredo Lanari". Universidad de Buenos Aires, Ciudad \\ Autónoma de Buenos Aires, Argentina.
}

Aviso de derechos de autor

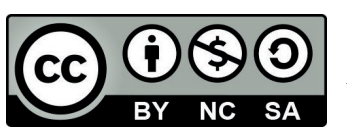

(C) 2021 Acta Gastroenterológica Latinoamericana. Este es un artículo de acceso abierto publicado bajo los términos de la Licencia Creative Commons Attribution (CC BY-NC-SA 4.0), la cual permite el uso, la distribución $y$ la reproducción de forma no comercial, siempre que se cite al autor y la fuente original.
Cite este artículo como: Cuzcano Villalobos B, Quinteros Figueroa K, Osada Liy J. Importancia de la clasificación de obesidad en la tasa de erradicación de Helicobacter pylori. Acta Gastroenterol Latinoam. 2021;51(1):7-9. https:/ldoi. org/10.52787/vfoy 2155

\section{Referencias}

1. Carabotti M, D’Ercole C, Iossa A, Corazziari E, Slecchia G, Severi C. Helicobacter pylori infection in obesity and its clinical outcome after bariatric surgery. World J Gastroenterology. 2014;20:647-53.

2. Lender N, Talley N, Enck P, Haag S, Zipfel S, Morrison M, Holtman J. Review article: associations between Helicobacter pylori and obesity-an ecological study. Alimentary Pharmacology and Therapeutics. 2014;40:24-31.

3. Lane JA, Murray LJ, Harvey IM. Randomised clinical trial: Helicobacter pylori eradication is associated with a significantly increased body mass index in a placebo-controlled study. Aliment Pharmacol Ther. 2011;33:922-9.

4. Osawa H. Ghrelin and Helicobacter pylori infection. World J Gastroenterology. 2008;14:6327-33.

5. Laudanno O, Thome M, Ahumarán G, Gollo P, González P, Mastruzzo M. Preoperative upper endoscopy in asymptomatic obese patients undergoing bariatric surgery: is it mandatory? Gastrointestinal endoscopy. 2019;89:554-5.

6. Pampillón N, Reynoso C, Pagano C. Consenso Argentino de Nutrición en Cirugía Bariátrica. Actualización en Nutrición. 2012;12:98-141.

7. Laudanno O, Ahumarán G, Thomé M, Gollo P, Khoury M, Gonzalez P. Tailored Helicobacter pylori eradication therapy in obese patients undergoing bariatric surgery. Revista Española de Enfermedades Digestivas. 2020. 